\title{
Sea quark contributions to the electromagnetic form factors of $\Sigma$ hyperons
}

\author{
Mingyang Yang $\odot^{*}$ and Ping Wang $\oplus^{\dagger}$ \\ Institute of High Energy Physics, CAS, P. O. Box 918(4), Beijing 100049, China \\ and College of Physics Sciences, University of Chinese Academy of Sciences, Beijing 100049, China
}

(Received 26 October 2021; accepted 13 December 2021; published 6 January 2022)

\begin{abstract}
We study the sea quark contributions to the electromagnetic form factors of $\Sigma$ baryons with nonlocal chiral effective theory. Both octet and decuplet intermediate states are included in the one loop calculation. $G_{\Sigma^{-}}^{u}$ and $G_{\Sigma^{+}}^{d}$ could be priority observables for the examination of sea quark contributions to the baryon structure because these quantities are much larger than the strange form factors of nucleons. It will be less difficult for the lattice simulation to determine the sign of these pure sea quark contributions unambiguously. In $\Sigma^{0}$, the light sea quark form factors $G_{\Sigma^{0}}^{u}$ and $G_{\Sigma^{0}}^{d}$ are identical. Since the light sea quark form factors in protons are different, it will be more meaningful to compare the lattice result of the light sea quark form factors in $\Sigma^{0}$ with that obtained from an effective field theory.
\end{abstract}

DOI: 10.1103/PhysRevD.105.016006

\section{INTRODUCTION}

Electromagnetic form factors of nucleons are among the most important observables of these building blocks of the ordinary matter in the Universe. A lot of theoretical and experimental efforts have been made for many years to get the precise values of the form factors. The form-factor data have triggered much activity in the determination of the flavor separated form factors of the dressed up, down, and strange quarks in the nucleon. It is well known that a complete characterization of the baryon substructure must go beyond three valence quarks. In particular, the strange quark contribution to the nucleon electromagnetic form factors is of special interest because it is purely from the sea quark. The strange form factors of the proton $G_{E}^{s}$ and $G_{M}^{s}$ have been obtained by a number of groups, such as SAMPLE at Bates [1], A4 at Mainz [2,3], and G0 [4,5] and HAPPEX [6-9] at Jefferson Lab, etc. However, up to now, the experiments are not able to determine the signs of $G_{E}^{s}$ and $G_{M}^{s}$ unambiguously although the global analyses do tend to suggest that $G_{M}^{s}<0$ is favored [10,11].

Theoretically, strange form factors of nucleons were investigated in various phenomenological models that give different predictions [12-22]. They were also studied in chiral perturbation theory (ChPT) or effective field theory (EFT) [23-25]. Historically, most formulations of ChPT are

\footnotetext{
*yangmy@ihep.ac.cn

pwang4@ihep.ac.cn
}

Published by the American Physical Society under the terms of the Creative Commons Attribution 4.0 International license. Further distribution of this work must maintain attribution to the author(s) and the published article's title, journal citation, and DOI. Funded by SCOAP ${ }^{3}$. based on the dimensional or the infrared regularization. Though ChPT is a successful and systematic approach, for the nucleon electromagnetic form factors, it is only valid for $Q^{2}<0.1 \mathrm{GeV}^{2}$ [26]. When vector mesons are included, the results are close to the experiments with $Q^{2}$ less than $0.4 \mathrm{GeV}^{2}$ [27]. Because of the unknown low energy constants appearing in the chiral Lagrangian, the capacity to predict the strange form factors is much limited. In other words, the strangeness vector current matrix elements that one wants to predict are the same quantities one needs to know in order to make such predictions. [23,25].

An alternative regularization method, namely the finiterange regularization (FRR), has been proposed. Inspired by quark models that account for the finite size of the nucleon as the source of the pion cloud, effective field theory with FRR has been widely applied to extrapolate the vector meson masses, magnetic moments, magnetic form factors, strange form factors, charge radii, first moments of GPDs, and the nucleon spin, etc. [28-30]. With the regulators, the loop integrals are convergent. One advantage of ChPT with the FRR is that strange form factors can be predicted with the assumption that there is no valence contribution from the tree level. No low energy constants are needed to cancel the loop divergence for predicting the strange form factors [31,32].

In recent years, we proposed a nonlocal chiral effective theory, which makes it possible to study the form factors at relatively large $Q^{2}$ [33-35]. With the introduction of the gauge links, the nonlocal Lagrangians are locally gauge invariant. The nonlocal interaction generates both the relativistic regulators, which make the loop integrals convergent, and the $Q^{2}$ dependence of form factors at tree level. The obtained electromagnetic form factors and strange form factors of nucleon are very close to both experimental data 
and lattice results $[33,34]$. This nonlocal EFT was further applied to study the parton distribution functions [36,37]. For the sea quark form factors of proton, the light sea quark contributions obtained in nonlocal EFT are quite different from the lattice results, though the strange quark contributions are comparable with each other [38-40]. There is an obvious flavor asymmetry of the $u$ sea and $d$ sea in nonlocal EFT, while the light sea quark contributions are the same for $u$ and $d$ in the lattice simulation.

Therefore, to get a better understanding of the sea quark properties, in this paper, we investigate the sea quark contributions to the electromagnetic form factors of $\Sigma$ hyperons. For $\Sigma^{+}\left(\Sigma^{-}\right)$, the $d(u)$ quark contribution is purely from the sea, which is the same as the $s$ quark contribution in nucleon. However, because the $d(u)$ quark contribution in $\Sigma^{+}\left(\Sigma^{-}\right)$comes from the $\pi$ meson loop, it is much larger than the strange quark contribution in the nucleon, concerning which the latter comes from the $K$ meson loop. It will be easier to get an unambiguous number for the lattice simulations, in spite of the statistic errors. For the experimentalists, it may also be possible to determine the signs of the light sea quark contributions in $\Sigma$ hyperons, since the magnetic moments of $\Sigma^{+}$and $\Sigma^{-}$have been measured precisely for many years. The investigation of the $d(u)$ quark contribution to $\Sigma^{+}\left(\Sigma^{-}\right)$form factors will definitely shed light on the signs of nucleon strange form factors. As pointed in Ref. [22], the signs of the strange form factors will indicate the possible configuration of $u u d s \bar{s}$ components of the proton. It could inform us whether the sea quark is in the baryon-meson configuration, or in the diquark configuration.

The flavor asymmetries of light sea quark will be also obvious in $\Sigma^{+}$and $\Sigma^{-}$as those in proton. We hope further lattice simulations could distinguish these sea quark asymmetries. To make the comparison between lattice simulation and nonlocal EFT meaningful, we calculate the light sea quark contributions in $\Sigma^{0}$ as well, where the $u$ sea and $d$ sea do have the same contributions. In this case, one can make a direct comparison between these two calculations. This paper is organized in the following way. The nonlocal Lagrangian is introduced in Sec. II. Numerical results will be discussed in Sec. III. Section IV is a short summary. The loop expressions of sea quark contributions are presented in the Appendix.

\section{NONLOCAL EFFECTIVE LAGRANGIAN}

The lowest order chiral Lagrangian involving baryons, pseudoscalar mesons, and their interactions can be written as $[33,37,41,42]$

$$
\begin{aligned}
\mathcal{L}= & i \operatorname{Tr} \bar{B}\left(\gamma_{\mu} \mathcal{D}^{\mu}-m_{B}\right) B+\bar{T}_{\mu}^{a b c}\left(i \gamma^{\mu \nu \alpha} \mathcal{D}_{\alpha}-m_{T} \gamma^{\mu \nu}\right) T_{\nu}^{a b c}+\frac{f^{2}}{4} \operatorname{Tr}\left[\mathcal{D}_{\mu} U\left(\mathcal{D}^{\mu} U\right)^{\dagger}\right] \\
& +D \operatorname{Tr}\left(\bar{B} \gamma_{\mu} \gamma_{5}\left\{A^{\mu}, B\right\}\right)+F \operatorname{Tr}\left(\bar{B} \gamma_{\mu} \gamma_{5}\left[A^{\mu}, B\right]\right)+\frac{\mathcal{C}}{f} \varepsilon^{a b c} \bar{T}_{\mu, a}^{d e}\left(g^{\mu \nu}+z \gamma^{\mu} \gamma^{\nu}\right) B_{c e} \mathcal{D}_{\nu} \phi_{b d}+\text { H.c. },
\end{aligned}
$$

where $D, F$, and $\mathcal{C}$ are the coupling constants and $z$ is the off shell parameter. $\phi$ is the matrices of pseudoscalar meson fields, $B$ is the matrix of octet baryons, $T$ is the fully symmetric tensor for decuplet baryons, and $\mathcal{A}_{\mu}$ represents the photon field. In the meson sector, the chiral covariant derivative is defined as $\mathcal{D}_{\mu} U=\partial_{\mu} U-i e \mathcal{A}_{\mu}\left[\lambda_{q}, U\right]$, while in the octet baryon sector, $\mathcal{D}_{\mu}$ is defined as $\mathcal{D}_{\mu} B=\partial_{\mu} B+\left[\Gamma_{\mu}, B\right]$. The pseudoscalar meson octet couples to the baryon field, via the vector and axial-vector combinations, respectively,

$$
\begin{aligned}
& \Gamma_{\mu}=\frac{1}{2}\left(\zeta \partial_{\mu} \zeta^{\dagger}+\zeta^{\dagger} \partial_{\mu} \zeta\right)+\frac{1}{2} i e \mathcal{A}_{\mu}\left(\zeta^{\dagger} \lambda_{q} \zeta+\zeta \lambda_{q} \zeta^{\dagger}\right), \\
& A_{\mu}=\frac{i}{2}\left(\zeta \partial_{\mu} \zeta^{\dagger}-\zeta^{\dagger} \partial_{\mu} \zeta\right)-\frac{1}{2} e \mathcal{A}_{\mu}\left(\zeta \lambda_{q} \zeta^{\dagger}-\zeta^{\dagger} \lambda_{q} \zeta\right),
\end{aligned}
$$

where

$$
\zeta^{2}=U=\exp \left(\frac{2 i \phi}{f}\right), \quad f=93 \mathrm{MeV} .
$$

In the decuplet sector, $\mathcal{D}_{\nu}$ is defined as $\mathcal{D}_{\nu} T_{\mu}^{a b c}=$ $\partial_{\nu} T_{\mu}^{a b c}+\left(\Gamma_{\nu}, T_{\mu}\right)^{a b c}, \quad$ with $\quad\left(\Gamma_{\nu}, T_{\mu}\right)^{a b c}=\left(\Gamma_{\nu}\right)_{d}^{a} T_{\mu}^{d b c}+$ $\left(\Gamma_{\nu}\right)_{d}^{b} T_{\mu}^{a d c}+\left(\Gamma_{\nu}\right)_{d}^{c} T_{\mu}^{a b d}$, where $\gamma^{\mu \nu \alpha}$ and $\gamma^{\mu \nu}$ are the antisymmetric matrices expressed as

$$
\gamma^{\mu \nu}=\frac{1}{2}\left[\gamma^{\mu}, \gamma^{\nu}\right], \quad \gamma^{\mu \nu \rho}=\frac{1}{4}\left\{\left[\gamma^{\mu}, \gamma^{\nu}\right], \gamma^{\rho}\right\} .
$$

In the above equations, to calculate the contributions to the form factors from each quark with unit charge, the matrices $\lambda_{q}$ are introduced as

$$
\lambda_{u}=\left(\begin{array}{lll}
1 & 0 & 0 \\
0 & 0 & 0 \\
0 & 0 & 0
\end{array}\right), \quad \lambda_{d}=\left(\begin{array}{lll}
0 & 0 & 0 \\
0 & 1 & 0 \\
0 & 0 & 0
\end{array}\right), \quad \lambda_{s}=\left(\begin{array}{lll}
0 & 0 & 0 \\
0 & 0 & 0 \\
0 & 0 & 1
\end{array}\right) .
$$

The total form factors can be obtained by replacing $\lambda_{q}$ with the charge matrix $Q_{q}=\operatorname{diag}(2 / 3,-1 / 3,-1 / 3)$ expressed as $Q_{q}=\frac{2}{3} \lambda_{u}-\frac{1}{3} \lambda_{d}-\frac{1}{3} \lambda_{s}$.

The octet, decuplet, and octet-decuplet transition operators for magnetic interactions are needed in the one loop 
calculations. The anomalous magnetic Lagrangian of octet baryons is written as

$$
\begin{aligned}
\mathcal{L}_{\text {oct }}= & \frac{e}{4 m_{B}}\left[c_{1} \operatorname{Tr}\left(\bar{B} \sigma^{\mu \nu}\left\{\mathcal{F}_{\mu \nu}^{+}, B\right\}\right)+c_{2} \operatorname{Tr}\left(\bar{B} \sigma^{\mu \nu}\left[\mathcal{F}_{\mu \nu}^{+}, B\right]\right)\right. \\
& \left.+c_{3} \operatorname{Tr}\left(\bar{B} \sigma^{\mu \nu} B\right) \operatorname{Tr}\left(\mathcal{F}_{\mu \nu}^{+}\right)\right]
\end{aligned}
$$

where

$$
\mathcal{F}_{\mu \nu}^{+}=-\frac{1}{2} \mathcal{F}_{\mu \nu}\left(\zeta^{\dagger} \lambda_{q} \zeta+\zeta \lambda_{q} \zeta^{\dagger}\right)
$$

The above Lagrangian will contribute to the Pauli form factor $F_{2}^{f, \Sigma}$, which is defined in Eq. (18). At the lowest order, the contribution of quark $q$ with unit charge to the octet magnetic moments, can be obtained. After expansion of the above equations, it is found that, taking $\Sigma$ hyperons as an example,

$F_{2}^{u, \Sigma^{+}}=c_{1}+c_{2}+c_{3}, \quad F_{2}^{d, \Sigma^{+}}=c_{1}-c_{2}+c_{3}, \quad F_{2}^{s, \Sigma^{+}}=c_{3}$, $F_{2}^{u, \Sigma^{-}}=c_{1}-c_{2}+c_{3}, \quad F_{2}^{d, \Sigma^{-}}=c_{1}+c_{2}+c_{3}, \quad F_{2}^{s, \Sigma^{-}}=c_{3}$.

Comparing with the results of the constituent quark model, where $F_{2}^{d, \Sigma^{+}}=0$, and $F_{2}^{u, \Sigma^{-}}=0$, we get

$$
c_{3}=c_{2}-c_{1} \text {. }
$$

The above relationship is consistent with that there is no strange quark contribution in bare nucleon.

The magnetic moment operators of decuplet and octetdecuplet transition are expressed as

$$
\mathcal{L}_{\mathrm{dec}}=-\frac{i e F_{2}^{T}}{4 M_{T}} \bar{T}_{\mu, a b c} \sigma_{\rho \lambda} F^{\rho \lambda} T^{\mu, a b c}
$$

and

$$
\begin{aligned}
\mathcal{L}_{\text {trans }}= & \frac{i e \mu_{T}}{4 m_{B}} F_{\mu \nu}\left(\epsilon^{i j k} \lambda_{q, i l} \bar{B}_{j m} \gamma^{\mu} \gamma_{5} T^{\nu, k l m}\right. \\
& \left.+\epsilon^{i j k} \lambda_{q, l i} \bar{T}^{\mu, k l m} \gamma^{\nu} \gamma_{5} B_{m j}\right),
\end{aligned}
$$

respectively.

The gauge invariant nonlocal Lagrangian can be obtained using the method in $[33,34,43]$. For instance, the local interaction between $\Sigma$ hyperons and $\pi^{+}$meson can be written as

$$
\mathcal{L}_{\Sigma \pi^{+}}^{\text {local }}=\frac{-F}{f} \bar{\Sigma}^{+}(x) \gamma^{\mu} \gamma_{5} \Sigma^{0}(x)\left(\partial_{\mu}+i e \mathcal{A}_{\mu}(x)\right) \pi^{+}(x) .
$$

The corresponding nonlocal Lagrangian follows,

$$
\begin{aligned}
\mathcal{L}_{\Sigma \pi^{+}}^{\mathrm{nl}}= & \frac{-F}{f} \int \mathrm{d}^{4} y \bar{\Sigma}^{+}(x) \gamma^{\mu} \gamma_{5} \Sigma^{0}(x) \\
& \times\left(\partial_{\mu}+i e \int \mathrm{d}^{4} a \mathcal{A}_{\mu}(x-a) F(a)\right) F(x-y) \\
& \times \exp \left[i e \int_{x}^{y} \mathrm{~d} z_{\nu} \int \mathrm{d}^{4} b \mathcal{A}^{\nu}(z-b) F(b)\right] \pi^{+}(y),
\end{aligned}
$$

where $F(x-y)$ is the correlation function. From the Lagrangian, one can see that the baryon fields are located at $x$, while the meson and photon fields are displaced. To make the Lagrangian locally gauge invariant, the gauge link $\exp \left[i e \int_{x}^{y} \mathrm{~d} z_{\nu} \int \mathrm{d}^{4} b \mathcal{A}^{\nu}(z-b) F(b)\right]$ is introduced. Therefore, the photon could be emitted or annihilated from either the minimal substitution term or the gauge link term. The correlation functions are associated with each photon field and meson field. With the correlation function, the regulator and momentum dependence of form factors at tree level can be generated automatically. In the numerical calculation, the correlation function in momentum space is chosen to be a dipole form.

The nonlocal baryon-photon interactions are obtained in the same procedure. For example, the local interaction between $\Sigma^{+}$and photon is written as

$$
\begin{aligned}
\mathcal{L}_{\mathrm{EM}}^{\text {local }}= & -e \bar{\Sigma}^{+}(x) \gamma^{\mu} \Sigma^{+}(x) \mathcal{A}_{\mu}(x) \\
& +\frac{\left(c_{1}+3 c_{2}\right) e}{12 m_{\Sigma}} \bar{\Sigma}^{+}(x) \sigma^{\mu \nu} \Sigma(x)^{+} \mathcal{F}_{\mu \nu}(x) .
\end{aligned}
$$

The corresponding nonlocal Lagrangian is expressed as

$$
\begin{aligned}
\mathcal{L}_{\mathrm{EM}}^{\mathrm{nl}}= & -e \int \mathrm{d}^{4} a \bar{\Sigma}^{+}(x) \gamma^{\mu} \Sigma(x)^{+} \mathcal{A}_{\mu}(x-a) F_{1}(a) \\
& +\frac{\left(c_{1}+3 c_{2}\right) e}{6} \int \mathrm{d}^{4} a \bar{\Sigma}^{+}(x) \\
& \times \frac{\sigma^{\mu \nu}}{2 m_{\Sigma}} \Sigma(x)^{+} \mathcal{F}_{\mu \nu}(x-a) F_{2}(a),
\end{aligned}
$$

where $F_{1}(a)$ and $F_{2}(a)$ are the correlation functions for the nonlocal electric and magnetic interactions.

The momentum dependence of the form factors at tree level can be easily obtained with the Fourier transformation of the correlation function. As in our previous work $[33,34]$, the correlation function is chosen such that the charge and magnetic form factors at tree level have the same momentum dependence as the baryon-meson vertex, i.e., $G_{M}^{B \text {,tree }}(q)=\mu_{B} G_{E}^{B \text {,tree }}(q)=\mu_{B} \tilde{F}(q)$, where $\tilde{F}(q)$ is the Fourier transformation of the correlation function $F(a)$. Therefore, the corresponding functions $\tilde{F}_{1}(q)$ and $\tilde{F}_{2}(q)$ of $\Sigma^{+}$, for example, are expressed as 
$\tilde{F}_{1}^{\Sigma^{+}}(q)=\tilde{F}(q) \frac{12 m_{\Sigma}^{2}+\left(3+c_{1}+3 c_{2}\right) Q^{2}}{3\left(4 m_{\Sigma}^{2}+Q^{2}\right)}$,

$\tilde{F}_{2}^{\Sigma^{+}}(q)=\tilde{F}(q) \frac{4\left(c_{1}+3 c_{2}\right) m_{\Sigma}^{2}}{3\left(4 m_{\Sigma}^{2}+Q^{2}\right)}$

where $Q^{2}=-q^{2}$ is the momentum transfer.

From Eq. (12), two kinds of $\Sigma-\pi$-photon vertices can be obtained. One is the normal coupling,

$$
\begin{aligned}
\mathcal{L}^{\text {norm }}= & -i e \frac{F}{f} \int \mathrm{d}^{4} y \bar{\Sigma}^{+}(x) \gamma^{\mu} \gamma_{5} \Sigma^{0}(x) \\
& \times \int \mathrm{d}^{4} a \mathcal{A}_{\mu}(x-a) F(a) F(x-y) \pi^{+}(y) .
\end{aligned}
$$

This interaction is similar to the traditional local Lagrangian except the correlation function. The other is the additional interaction, obtained by the expansion of the gauge link,

$$
\begin{aligned}
\mathcal{L}^{\text {add }}= & -i e \frac{F}{f} \int \mathrm{d}^{4} y \bar{\Sigma}^{+}(x) \gamma^{\mu} \gamma_{5} \Sigma^{0}(x) \partial_{x, \mu} \\
& \times\left[F(x-y) \int_{x}^{y} \mathrm{~d} z_{\nu} \int \mathrm{d}^{4} a \mathcal{A}^{\nu}(z-a) F(a) \pi^{+}(y)\right] .
\end{aligned}
$$

The additional interaction is crucial to guarantee the charge conservation. With the nonlocal Lagrangian above, we can calculate the electromagnetic form factors.
The contributions of individual quark flavor $f(f=u$, $d, s)$ to the Dirac and Pauli form factors of $\Sigma$ hyperons are defined as

$$
\begin{aligned}
& \left\langle\Sigma\left(p^{\prime}\right)\left|J_{\mu}^{f}\right| \Sigma(p)\right\rangle \\
& \quad=\bar{u}\left(p^{\prime}\right)\left\{\gamma_{\mu} F_{1}^{f, \Sigma}\left(Q^{2}\right)+\frac{i \sigma_{\mu \nu} q^{\nu}}{2 m_{\Sigma}} F_{2}^{f, \Sigma}\left(Q^{2}\right)\right\} u(p),
\end{aligned}
$$

where $q=p^{\prime}-p$. The electromagnetic form factors are defined as the combinations of the above form factors,

$$
\begin{aligned}
& G_{E}^{f, \Sigma}\left(Q^{2}\right)=F_{1}^{f, \Sigma}\left(Q^{2}\right)-\frac{Q^{2}}{4 m_{\Sigma}^{2}} F_{2}^{f, \Sigma}\left(Q^{2}\right), \\
& G_{M}^{f, \Sigma}\left(Q^{2}\right)=F_{1}^{f, \Sigma}\left(Q^{2}\right)+F_{2}^{f, \Sigma}\left(Q^{2}\right) .
\end{aligned}
$$

According to the Lagrangians, the one-loop Feynman diagrams that contribute to the $\Sigma$ electromagnetic form factors are shown in Fig. 1. The matrix element of Eq. (18) can be evaluated. The $\pi$ meson loops have the dominant contributions, while the contributions from $K$ meson loops are much smaller due to the large $K$ meson mass. The contributions from $\eta$ and $\eta^{\prime}$ loops are even smaller, which are neglected in our calculation. The inclusion of these mesons does not affect the main conclusion below. For each diagram in Fig. 1, there exist quenched and disconnected diagrams. In order to obtain the sea quark contribution, we need to find the coefficients for the disconnected diagrams.
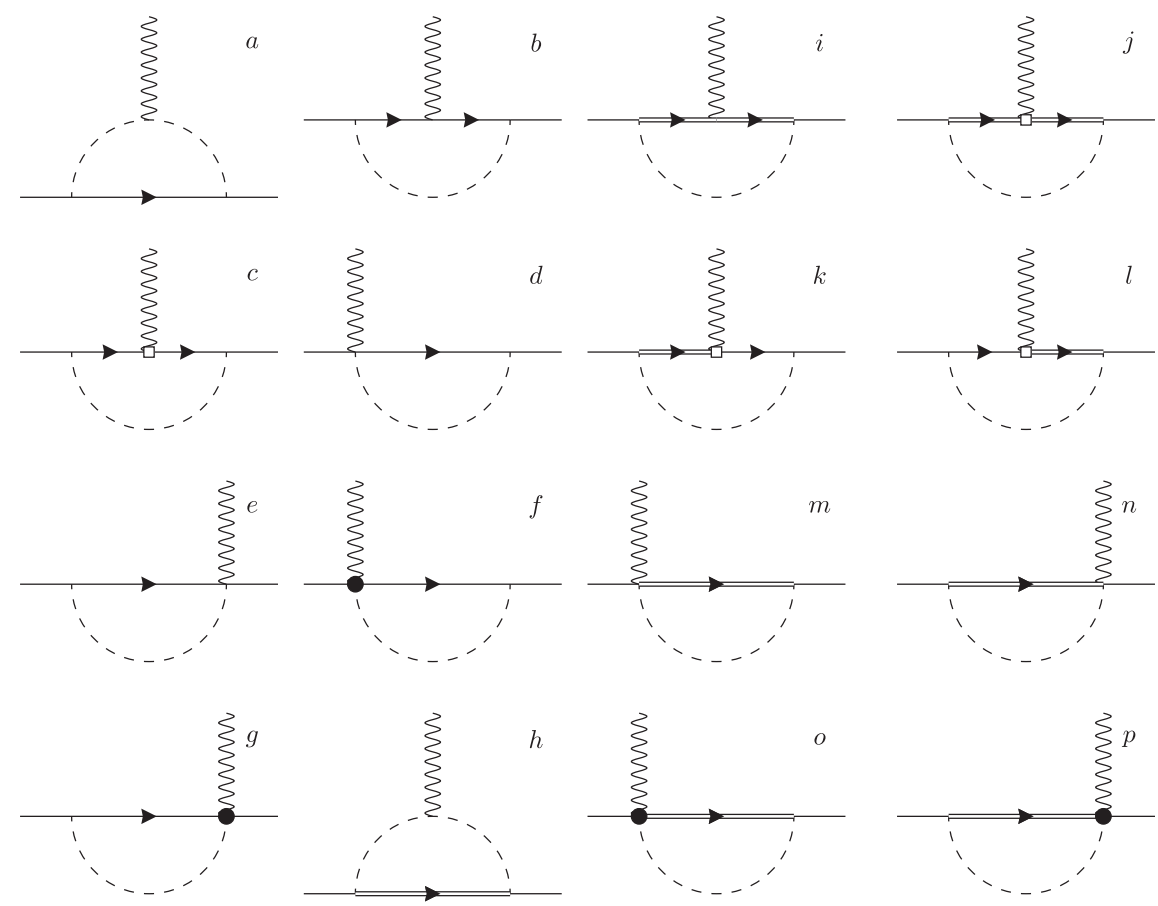

FIG. 1. One-loop Feynman diagrams for the hyperon electromagnetic form factors. The solid, double-solid, dashed, and wave lines are for the octet baryons, decuplet baryons, pseudoscalar mesons, and photons, respectively. The rectangle and black dot represent magnetic and additional interacting vertex. 


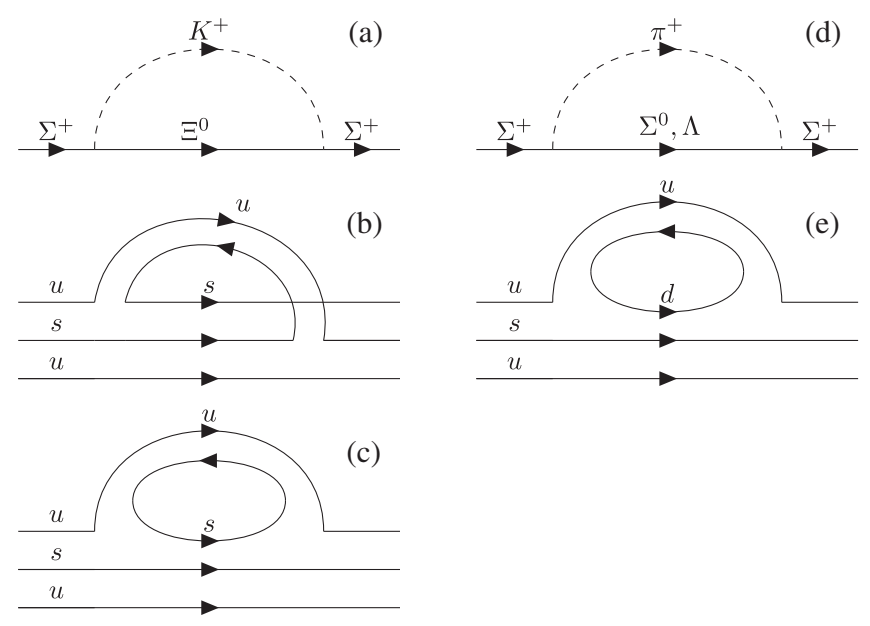

FIG. 2. Quark flow diagrams for $K^{+}$and $\pi^{+}$. (a) is the quenched diagram for $K^{+}$. (b) and (c) are the disconnected sea diagrams for $K^{+}$and $\pi^{+}$, respectively.

The coefficients for the quenched and disconnected loop diagrams can be extracted separately as in Ref. [44], using the quark flows of Fig. 2. The obtained coefficients are the same as those extracted within the graded symmetry formalism in quenched chiral perturbation theory [45].

Here, as an example, we show how to separate the quenched and sea contributions of strange quark to $\Sigma^{+}$in the intermediate $\Xi^{0}$ rainbow diagram. In Fig. 2, the quarkflow diagrams and the corresponding Feynman diagrams are plotted. The strange quark contribution of Fig. 2(a) includes the quenched [Fig. 2(b)] and sea [Fig. 2(c)] contributions, while the strange quark contribution of Fig. 2(d) is only from the sea diagram Fig. 2(e). The coefficient of Fig. 2(a) for the total contribution is $(D+F)^{2} / 2 f^{2}$, which is the sum of the coefficients of Figs. 2(b) and 2(c). Due to the SU(3) symmetry, the coefficient of the sea contribution of Fig. 2(c) is the same as that of Fig. 2(e), where the $s$-quark loop is replaced by the $d$-quark loop. The coefficient of Fig. 2(e) can be obtained by summing the coefficients for the intermediate $\Sigma^{0}$ and $\Lambda$ states, which is $\left(D^{2}+3 F^{2}\right) / 3 f^{2}$. Therefore, subtracting the coefficient of Fig. 2(c) from that of Fig. 2(a), we can get the coefficient for the quenched diagram Fig. 2(b), which is $\left(D^{2}+6 D F-3 F^{2}\right) / 6 f^{2}$. With the same method, all the quenched and sea contributions of $u, d$, and $s$ quarks can be obtained and the coefficients are listed in Table I.

With the obtained coefficients for the sea quark diagrams, the sea quark form factors of $u, d$, and $s$ in $\Sigma$ hyperons can be evaluated by summing up all the contributions in Fig. 1. The expressions for the intermediate octet and decuplet baryons of each diagram are written in the Appendix. In the next section, we will discuss the numerical results.
TABLE I. The coefficients of $u, d$, and $s$ quark in $\Sigma$, for both quenched and sea quark-flow diagrams.

\begin{tabular}{lcccc}
\hline \hline Configurations & $\Sigma-\pi$ & $\Lambda-\pi$ & $N-K$ & $\Xi-K$ \\
\hline$\Sigma_{u, \text { quench }}^{+}$ & & & & $-\frac{D^{2}+6 D F-3 F^{2}}{6 f^{2}}$ \\
$\Sigma_{u, \text { sea }}^{+}$ & $\frac{D^{2}+3 F^{2}}{6 f^{2}}$ & & & \\
$\Sigma_{d, \text { sea }}^{+}$ & $\frac{F^{2}}{f^{2}}$ & $\frac{D^{2}}{3 f^{2}}$ & $\frac{(D-F)^{2}}{2 f^{2}}$ & \\
$\Sigma_{u, \text { quench }}^{0}$ & & & $\frac{D^{2}-18 D F+9 F^{2}}{36 f^{2}}$ & $\frac{8 D^{2}-9(D+F)^{2}}{36 f^{2}}$ \\
$\Sigma_{u, \text { sea }}^{0}$ & $\frac{2 D^{2}}{9 f^{2}}$ & $\frac{D^{2}}{6 f^{2}}$ & $\frac{2 D^{2}}{9 f^{2}}$ & \\
$\Sigma_{d, \text { quench }}^{0}$ & & & $\frac{D^{2}-18 D F+9 F^{2}}{36 f^{2}}$ & $\frac{8 D^{2}-9(D+F)^{2}}{36 f^{2}}$ \\
$\Sigma_{d, \text { sea }}^{0}$ & $\frac{2 D^{2}}{9 f^{2}}$ & $\frac{D^{2}}{6 f^{2}}$ & $\frac{2 D^{2}}{9 f^{2}}$ & \\
$\Sigma_{s, \text { quench }}^{+}$ & & & & $\frac{D^{2}+6 D F-3 F^{2}}{6 f^{2}}$ \\
$\Sigma_{s, \text { sea }}^{+}$ & & & & $\frac{D^{2}+3 F^{2}}{3 f^{2}}$ \\
$\Sigma_{s, \text { quench }}^{0}$ & & & $-\frac{D^{2}-18 D F+9 F^{2}}{18 f^{2}}$ & $\frac{D^{2}+18 D F+9 F^{2}}{18 f^{2}}$ \\
$\Sigma_{s, \text { sea }}^{0}$ & & & & $\frac{4 D^{2}}{9 f^{2}}$ \\
\hline \hline
\end{tabular}

\section{NUMERICAL RESULTS}

The coupling constants between octet baryons and mesons are determined by two parameters $D$ and $F$. In the numerical calculations, the parameters are chosen to be $D=0.76$ and $F=0.50\left(g_{A}=D+F=1.26\right)$ [46]. The coupling constant $\mathcal{C}$ is chosen to be 1.0 , which is the same as in Refs. [33,34]. The off shell parameter $z$ is -1 [47]. Besides, the physical masses are used for mesons and octet and decuplet baryons. The covariant regulator is chosen to be a dipole form $[34,35]$

$$
\tilde{F}(k)=\frac{\Lambda^{4}}{\left(k^{2}-m_{j}^{2}-\Lambda^{2}\right)^{2}},
$$

where $m_{j}$ is the meson mass for the baryon-meson interaction, and it is zero for the hadron-photon interaction. It was found that when $\Lambda$ is around $0.9 \mathrm{GeV}$, the obtained electromagnetic and strange form factors were very close to the experimental data and lattice simulation. The low energy constants $c_{1}$ and $c_{2}$ are determined by fitting the experimental magnetic moments of octet baryons. They are found to be 1.736 and 0.329 , which give the minimal $\chi^{2}$ of the octet magnetic moments.

The sea quark contributions to the magnetic form factor of $\Sigma^{+}$baryon versus momentum transfer $Q^{2}$ are plotted in Fig. 3. The solid, dashed, and dotted lines are for $u, d$, and $s$ quark with unit charge, respectively. The lines colored red, green, and blue are for $\Lambda=0.8,0.9$, and $1.0 \mathrm{GeV}$, respectively. To obtain an impression on the order of magnitude of the sea quark contributions, the total magnetic form factor of $\Sigma^{+}$calculated within the same framework is also plotted at the corner. The total $\mu_{\Sigma^{+}}$is about 2.4, which is consistent with the experimental 


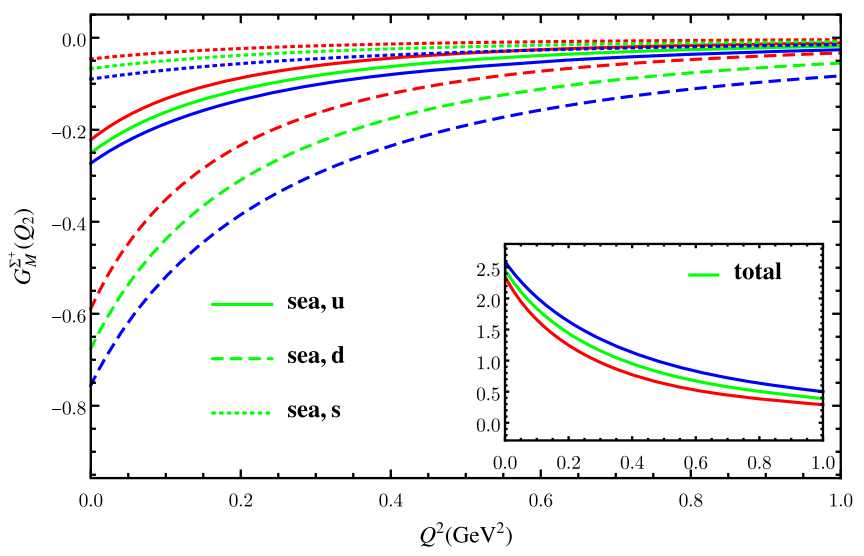

FIG. 3. The sea quark contributions to the magnetic form factors of $\Sigma^{+}$hyperon versus momentum transfer $Q^{2}$. The solid, dashed, and dotted lines are for the contributions from $u$ sea, $d$ sea, and $s$ sea, respectively. The lines with color red, green, and blue are for $\Lambda=0.8,0.9$, and $1.0 \mathrm{GeV}$, respectively. The small figure at the corner is for the total magnetic form factor of $\Sigma^{+}$.

value [48]. From the figure, one can see that all the sea quark contributions to the magnetic form factors of $\Sigma^{+}$are negative. When $Q^{2}=0$, the sea quark magnetic moments $\mu^{u, \Sigma^{+}}, \mu^{d, \Sigma^{+}}$, and $\mu^{s, \Sigma^{+}}$are $-0.251 \pm 0.025$, $-0.676 \pm 0.083$, and $-0.067 \pm 0.022$, respectively. They remain negative, and their magnitudes decrease with the increasing momentum transfer. Compared with the sea quark contribution in the nucleon, $G_{M}^{f, \Sigma^{+}}$is larger than the corresponding $G_{M}^{f, N}$. It is expected the light quark contribution is much larger than the strange quark contribution, as in the nucleon case. The magnitude of $G_{M}^{d, \Sigma^{+}}$is the largest one, and it is much larger than $G_{M}^{u, \Sigma^{+}}$. Similarly as in the nucleon, the asymmetry of $u$ sea and $d$ sea is obvious. This flavor asymmetry is not raised by the mass difference of $u$ and $d$ quarks. It is because of the different numbers of valence quarks $u$ and $d$ in $\Sigma^{+}$. For example, in Fig. 1, there is only $\pi^{+}$loop that contributes to the form factor of the $d$ sea quark. No $\pi^{-}$diagram exists which contributes to the form factor of $u$ sea quark. It is interesting to see whether this sea quark asymmetry could be obtained from lattice simulation. Currently, lattice simulations have not shown this asymmetry in proton, where the light quark form factors are somewhat like the average of the $u$ and $d$ contributions. Since the difference between $u$ sea and $d$ sea is very large, to make the direct comparison between EFT calculation and lattice simulation meaningful, we will show the light sea quark contributions to the form factors of $\Sigma^{0}$ hyperon latter, where the sea contributions from $u$ and $d$ quark are the same.

In the $\Sigma^{+}$hyperon, there is no valence $d$ quark. All the contributions of the $d$ quark is from the sea. This is comparable with the strange quark contribution in nucleon. The difference is that $G_{M}^{d, \Sigma^{+}}$is generated from the $\pi$ loop, while $G_{M}^{s, N}$ is from the $K$ loop. As a result, $G_{M}^{d, \Sigma^{+}}$is much

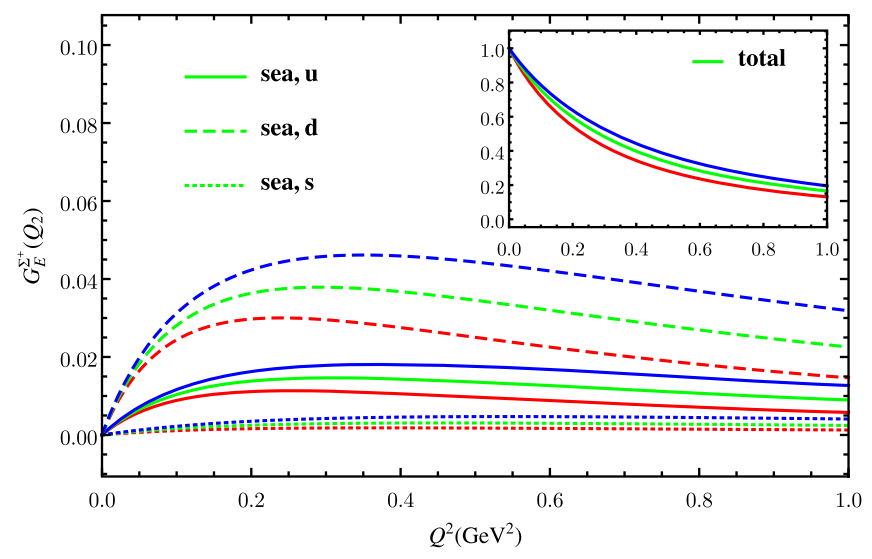

FIG. 4. Same as Fig. 3 but for the electric form factors.

larger than the strange magnetic form factor of the nucleon, which makes it easy for lattice simulation to get an unambiguous sign for this pure sea quark contribution. If lattice confirms that $G_{M}^{d, \Sigma^{+}}$is negative, then it will suggest the meson-loop scenario; i.e., the antiquark in the nucleon should be in the state with orbital angular momentum $1[22]$.

The sea quark contributions to the electric form factor of $\Sigma^{+}$hyperon with the same choice of $\Lambda$, are plotted in Fig. 4 . The total electric form factor of $\Sigma^{+}$is plotted in the inset as well. Because the contributions of quark and antiquark are from the sea, their electric form factors are zero at $Q^{2}=0$. The additional diagrams are important to get $G_{E}^{f, \Sigma}(0)=0$. They are generated from the additional interaction, which guarantees the local gauge invariance. All the electric form factors of sea quark increase first, and then decrease with the increasing $Q^{2}$. Again, there is a large sea quark asymmetry between $G_{E}^{u, \Sigma^{+}}$and $G_{E}^{d, \Sigma^{+}} . G_{E}^{d, \Sigma^{+}}$is more than twice bigger than $G_{E}^{u, \Sigma^{+}}$. When $Q^{2}$ is around $0.3 \mathrm{GeV}, G_{E}^{d, \Sigma^{+}}$ is about $0.038 \pm 0.008$. Compared with the strange electric form factor, $G_{E}^{d, \Sigma^{+}}$is about 10 times larger. Therefore, for both electric and magnetic form factors, $G_{E}^{d, \Sigma^{+}}$and $G_{M}^{d, \Sigma^{+}}$ are good physical observables for lattice simulation. The hyperon form factors would not only shed light on the structure of hyperons, but also provide information on nucleon. Since the contributions of $u, d$, and $s$ quark with unit charge in $\Sigma^{-}$are the same as $d, u$, and $s$ quark contributions in $\Sigma^{+}$, we will not show the results for $G_{E(M)}^{f, \Sigma^{-}}$.

Now, we discuss the sea quark contributions to the form factors of $\Sigma^{0}$. As we have pointed out, in nucleon or $\Sigma^{+}$, there is a large asymmetry between $u$ sea and $d$ sea. However, in $\Sigma^{0}$, the sea quark contributions from $u$ and $d$ are the same if we ignore the mass difference between them. We can make a direct comparison of the light sea quark form factors between lattice simulation and EFT. The total and sea quark contributions to the magnetic form factor of $\Sigma^{0}$ hyperon versus momentum transfer $Q^{2}$ are 


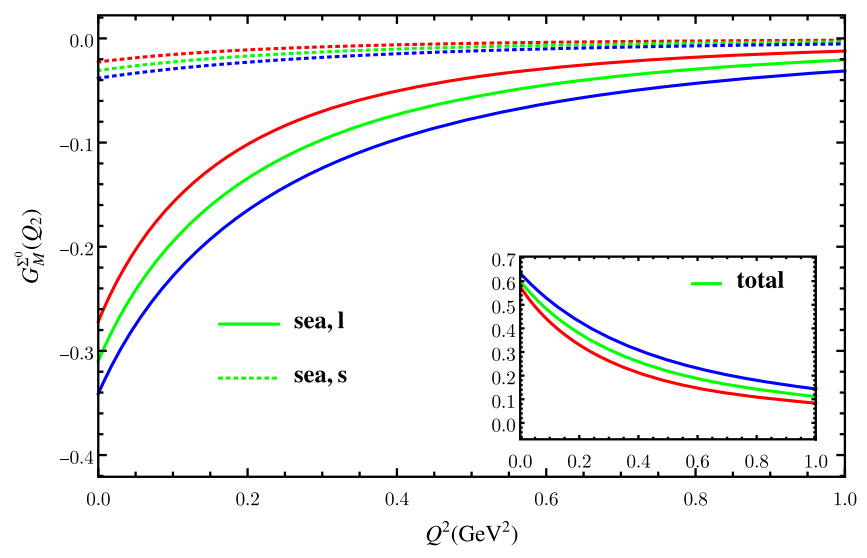

FIG. 5. The sea quark contributions to the magnetic form factors of $\Sigma^{0}$ hyperon versus momentum transfer $Q^{2}$. The solid and dashed lines are for the contributions from light sea and $s$ sea, respectively. The lines with color red, green, and blue are for $\Lambda=0.8,0.9$, and $1.0 \mathrm{GeV}$, respectively. The inset is for the total magnetic form factor of $\Sigma^{0}$.

plotted in Fig. 5. From the figure one can see that the sea quark form factors are negative and the shape of the momentum dependence of $G_{M}^{f, \Sigma^{0}}$ is close to $G_{M}^{f, \Sigma^{+}}$. The sea contribution from strange quark in $\Sigma^{0}$ has a similar magnitude as that in the nucleon and $\Sigma^{+}$. For the light quark contribution, the magnitude of $G_{M}^{l, \Sigma^{0}}$ is slightly bigger than $G_{M}^{u, \Sigma^{+}}$and much smaller than $G_{M}^{d, \Sigma^{+}}$. At $Q^{2}=0$ with central-valued $\Lambda, \mu^{l, \Sigma^{0}}=-0.310$, and $\mu^{s, \Sigma^{0}}=-0.031$.

The total and sea quark contributions to the electric form factors of $\Sigma^{0}$ baryon are plotted in Fig. 6. As for the magnetic case, in $\Sigma^{0}, G_{E}^{u, \Sigma^{0}}=G_{E}^{d, \Sigma^{0}}=G_{E}^{l, \Sigma^{0}}$, and $G_{E}^{l, \Sigma^{0}}$ lies between $G_{E}^{u, \Sigma^{+}}$and $G_{E}^{d, \Sigma^{+}}$. When $Q^{2}=0.3 \mathrm{GeV}^{2}, G_{E}^{l, \Sigma^{0}} \sim$ 0.019 with central-valued $\Lambda$. For the sea quarks with unit charge, their electric form factors are all positive, no matter the total charge of the baryon is $1,-1$, or 0 . Here, the sea quark form factors from the quark pair are obtained from the meson loop. In the baryon-meson scenario, the baryon

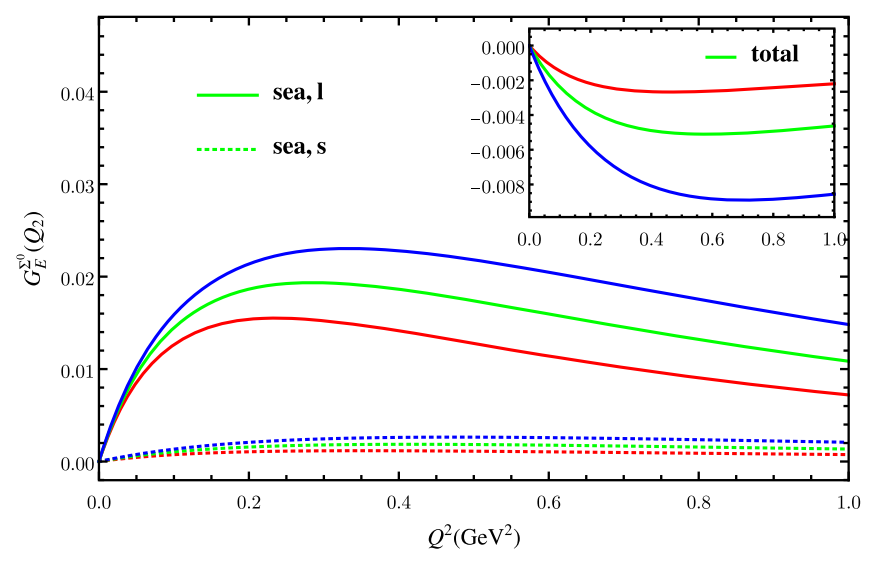

FIG. 6. Same as Fig. 5 but for the electric form factors. is surrounded by the meson where the charge of antiquark is -1 . The positive electric form factors of sea quark are consistent with this meson loop scenario.

With the calculated form factors, the contribution to the radii can be obtained as

$$
\left\langle r_{M(E)}^{2}\right\rangle=-\left.6 \frac{\mathrm{d} G_{M(E)}}{\mathrm{d} Q^{2}}\right|_{Q^{2}=0},
$$

where the magnetic form factors are not divided by the corresponding magnetic moments. Since all the sea quark form factors increase with the increasing $Q^{2}$ at low momentum transfer, the radii are all negative. The magnetic and charge radii of $d$ sea in $\Sigma^{+}$is larger than those of $u$ sea, and both of them are more than 10 times larger than the strange radii.

\section{SUMMARY}

The sea quark contributions to the electromagnetic form factors of $\Sigma$ hyperons are studied within the nonlocal chiral effective theory. Both the octet and decuplet intermediate states are included in our calculation. The correlation functions in the nonlocal Lagrangian make the loop integrals ultraviolet convergent. The gauge links guarantee that the nonlocal Lagrangians are locally gauge invariant. The expansion of the gauge links generates the additional diagrams and, as a result, the electric form factors of sea quarks are zero at $Q^{2}=0$. The obtained sea quark magnetic form factors of $u, d$ and $s$ are all negative, while the electric form factors are all positive. They are consistent with the scenario of baryon-meson configurations in dressed baryons. When $Q^{2}=0, \mu^{d, \Sigma^{+}}\left(\mu^{u, \Sigma^{-}}\right)$is much larger than the strange magnetic form factor $\mu^{s, N}$. For the charge form factors, $G_{E}^{d, \Sigma^{+}}\left(G_{E}^{u, \Sigma^{-}}\right)$is also much larger than $G_{E}^{s, N}$. Since there is no valence quark contributions to $G^{d, \Sigma^{+}}\left(G^{u, \Sigma^{-}}\right)$as $G^{s, N}, G^{d, \Sigma^{+}}\left(G^{u, \Sigma^{-}}\right)$could be a better physical observables for studying sea quark properties in baryons, for lattice simulations or possible experimental measurements. We also found there are large asymmetries of light sea quark form factors in charged $\Sigma$ hyperons. For both magnetic and electric form factors, the contributions of $d$ sea are significantly larger than those of $u$ sea. To make the direct comparison of light sea quark form factors between lattice simulation and EFT, we calculated the sea quark contributions in $\Sigma^{0}$. In this case, $G^{u, \Sigma^{0}}$ equals to $G^{d, \Sigma^{0}}$. The calculations of the sea quark form factors in hyperons, will not only shed light on the structure of hyperons, but also provide important information on nucleon structure. As a summary, we list the magnetic moments and radii of sea quarks in $\Sigma$ hyperons in Table II. The corresponding values for proton are also listed for comparison. 
TABLE II. The magnetic moments (in units of the nucleon magneton $\mu_{N}$ ), magnetic and charge radii of sea quarks in $\Sigma$ hyperons and proton.

\begin{tabular}{|c|c|c|c|c|c|c|c|c|c|}
\hline Baryon & $\mu_{\mathrm{sea}}^{u}$ & $\mu_{\text {sea }}^{d}$ & $\mu_{\text {sea }}^{s}$ & $\left\langle r_{M}^{2}\right\rangle_{\text {sea }}^{u}$ & $\left\langle r_{M}^{2}\right\rangle_{\text {sea }}^{d}$ & $\left\langle r_{M}^{2}\right\rangle_{\text {sea }}^{s}$ & $\left\langle r_{E}^{2}\right\rangle_{\text {sea }}^{u}$ & $\left\langle r_{E}^{2}\right\rangle_{\text {sea }}^{d}$ & $\left\langle r_{E}^{2}\right\rangle_{\text {sea }}^{S}$ \\
\hline$\Sigma^{+}$ & $-0.251 \pm 0.025$ & $-0.676 \pm 0.083$ & $-0.067 \pm 0.022$ & $-0.294 \pm 0.013$ & $-0.811 \pm 0.029$ & $-0.049 \pm 0.006$ & $-0.041 \pm 0.003$ & $-0.119 \pm 0.007$ & $-0.005 \pm 0.002$ \\
\hline$\Sigma^{0}$ & $-0.310 \pm 0.035$ & $-0.310 \pm 0.035$ & $-0.031 \pm 0.008$ & $-0.394 \pm 0.015$ & $-0.394 \pm 0.015$ & $-0.024 \pm 0.002$ & $-0.061 \pm 0.003$ & $-0.061 \pm 0.003$ & $-0.003 \pm 0.001$ \\
\hline$p$ & $-0.111 \pm 0.005$ & $-0.375 \pm 0.045$ & $-0.037 \pm 0.011$ & $-0.142 \pm 0.011$ & $-0.418 \pm 0.018$ & $-0.026 \pm 0.003$ & $-0.036 \pm 0.002$ & $-0.074 \pm 0.005$ & $-0.004 \pm 0.001$ \\
\hline
\end{tabular}

\section{ACKNOWLEDGMENTS}

This work was supported by the National Natural Science Foundation of China under Grant No. 11975241.

\section{APPENDIX: LOOP EXPRESSIONS}

In this appendix, we show the expressions of sea quark contributions in $\Sigma^{+}$hyperon for each diagram. The contributions of Fig. 1(a) are written as

$$
\Gamma_{a, u}^{\mu}\left(\Sigma^{+}\right)=\frac{D^{2}+3 F^{2}}{6 f^{2}} I_{a}^{\mu, \Sigma \pi}
$$

$$
\Gamma_{a, d}^{\mu}\left(\Sigma^{+}\right)=\frac{D^{2}}{3 f^{2}} I_{a}^{\mu, \Lambda \pi}+\frac{F^{2}}{f^{2}} I_{a}^{\mu, \Sigma \pi}+\frac{(D-F)^{2}}{2 f^{2}} I_{a}^{\mu, N K},
$$

$\Gamma_{a, s}^{\mu}\left(\Sigma^{+}\right)=\frac{D^{2}+3 F^{2}}{3 f^{2}} I_{a}^{\mu, \Xi K}$,

where the integral $I_{a}^{\mu, B M}$ is expressed as

$$
I_{a}^{\mu, B M}=\bar{u}\left(p^{\prime}\right) \tilde{F}(q) \int \frac{\mathrm{d}^{4} k}{(2 \pi)^{4}} \frac{\tilde{F}(q+k) \tilde{F}(k)}{D_{M}(k+q)} \frac{-(2 k+q)^{\mu}}{D_{M}(k)}(\not k+q) \gamma_{5} \frac{1}{\not p-\not k-m_{B}} \not k \gamma_{5} u(p) .
$$

$D_{M}(k)$ is defined as

$$
D_{M}(k)=k^{2}-m_{M}^{2}+i \varepsilon .
$$

$m_{B}$ and $m_{M}$ are the masses of the intermediate octet baryon $B$ and meson $M$, respectively. The formulas for $\Sigma^{0}$ are similar but with different coefficients. The contributions of Fig. 1(b) are expressed as

$$
\begin{aligned}
\Gamma_{b, u}^{\mu}\left(\Sigma^{+}\right)= & \frac{D^{2}\left(\left(c_{1}+3\left(c_{2}-c_{1}\right)+3\right) Q^{2}+12 m_{\Sigma}^{2}\right)+6 c_{1} D F Q^{2}+9 F^{2}\left(\left(c_{2}+1\right) Q^{2}+4 m_{\Sigma}^{2}\right)}{18 f^{2}\left(4 m_{\Sigma}^{2}+Q^{2}\right)} I_{b}^{\mu, \Sigma \pi}, \\
\Gamma_{b, d}^{\mu}\left(\Sigma^{+}\right)= & \frac{D\left(\left(c_{1}+3\left(c_{2}-c_{1}\right)+3\right) D Q^{2}+3 c_{1} F Q^{2}+12 D m_{\Lambda}^{2}\right)}{9 f^{2}\left(4 m_{\Lambda}^{2}+Q^{2}\right)} I_{b}^{\mu, \Lambda \pi} \\
& +\frac{F\left(c_{1} D Q^{2}+3 F\left(\left(c_{2}+1\right) Q^{2}+4 m_{\Sigma}^{2}\right)\right)}{3 f^{2}\left(4 m_{\Sigma}^{2}+Q^{2}\right)} I_{b}^{\mu, \Sigma \pi}+\frac{(D-F)^{2}\left(\left(c_{2}-c_{1}\right) Q^{2}+4 m_{N}^{2}+Q^{2}\right)}{2 f^{2}\left(4 m_{N}^{2}+Q^{2}\right)} I_{b}^{\mu, N K}, \\
\Gamma_{b, s}^{\mu}\left(\Sigma^{+}\right)= & \frac{D^{2}\left(\left(c_{1}+3\left(c_{2}-c_{1}\right)+3\right) Q^{2}+12 m_{\Xi}^{2}\right)+6 c_{1} D F Q^{2}+9 F^{2}\left(\left(c_{2}+1\right) Q^{2}+4 m_{\Xi}^{2}\right)}{9 f^{2}\left(4 m_{\Xi}^{2}+Q^{2}\right)} I_{b}^{\mu, \Xi K},
\end{aligned}
$$

where the integral $I_{b}^{\mu, B M}$ is written as

$$
I_{b}^{\mu, B M}=\bar{u}\left(p^{\prime}\right) \tilde{F}(q) \int \frac{\mathrm{d}^{4} k}{(2 \pi)^{4}} \frac{\tilde{F}(k)^{2}}{D_{M}(k)} \not \gamma_{5} \frac{1}{\not p^{\prime}-\not k-m_{B}} \gamma^{\mu} \frac{1}{\not p-\not k-m_{B}} \not \gamma_{5} u(p) .
$$

Figure 1(c) is similar to Fig. 1(b), except the former is for the magnetic interaction. The contributions of this diagram are written as

$$
\Gamma_{c, u}^{\mu}\left(\Sigma^{+}\right)=\frac{i m_{\Sigma}\left(3\left(c_{2}-c_{1}\right)\left(D^{2}+3 F^{2}\right)+c_{1}(D+3 F)^{2}\right)}{9 f^{2}\left(4 m_{\Sigma}^{2}+Q^{2}\right)} I_{c}^{\mu, \Sigma \pi},
$$




$$
\begin{aligned}
\Gamma_{c, d}^{\mu}\left(\Sigma^{+}\right)= & \frac{2 i D m_{\Lambda}\left(c_{1}(D+3 F)+3\left(c_{2}-c_{1}\right) D\right)}{9 f^{2}\left(4 m_{\Lambda}^{2}+Q^{2}\right)} I_{c}^{\mu, \Lambda \pi}+\frac{2 i F m_{\Sigma}\left(c_{1}(D+3 F)+3\left(c_{2}-c_{1}\right) F\right)}{3 f^{2}\left(4 m_{\Sigma}^{2}+Q^{2}\right)} I_{c}^{\mu, \Sigma \pi} \\
& +\frac{i\left(c_{2}-c_{1}\right)(D-F)^{2} m_{N}}{f^{2}\left(4 m_{N}^{2}+Q^{2}\right)} I_{c}^{\mu, N K}, \\
\Gamma_{c, s}^{\mu}\left(\Sigma^{+}\right)= & \frac{2 i m_{\Xi}\left(3\left(c_{2}-c_{1}\right)\left(D^{2}+3 F^{2}\right)+c_{1}(D+3 F)^{2}\right)}{9 f^{2}\left(4 m_{\Xi}^{2}+Q^{2}\right)} I_{c}^{\mu, \Xi K},
\end{aligned}
$$

where $I_{c}^{\mu, B M}$ is

$$
I_{c}^{\mu, B M}=\bar{u}\left(p^{\prime}\right) \tilde{F}(q) \int \frac{\mathrm{d}^{4} k}{(2 \pi)^{4}} \frac{\tilde{F}(k)^{2}}{D_{M}(k)} \not k \gamma_{5} \frac{1}{\not p^{\prime}-\not k-m_{B}} i \sigma^{\mu \nu} q_{\nu} \frac{1}{\not p-\not k-m_{B}} \not k \gamma_{5} u(p) .
$$

Figures 1(d) and 1(e) are the Kroll-Ruderman diagrams. The contributions of these two diagrams are written as

$$
\begin{gathered}
\Gamma_{d+e, u}^{\mu}\left(\Sigma^{+}\right)=\frac{D^{2}+3 F^{2}}{6 f^{2}} I_{d+e}^{\mu, \Sigma \pi}, \\
\Gamma_{d+e, d}^{\mu}\left(\Sigma^{+}\right)=\frac{D^{2}}{3 f^{2}} I_{d+e}^{\mu, \Lambda \pi}+\frac{F^{2}}{f^{2}} I_{d+e}^{\mu, \Sigma \pi}+\frac{(D-F)^{2}}{2 f^{2}} I_{d+e}^{\mu, N K}, \\
\Gamma_{d+e, s}^{\mu}\left(\Sigma^{+}\right)=\frac{D^{2}+3 F^{2}}{3 f^{2}} I_{d+e}^{\mu, \Xi K},
\end{gathered}
$$

where

$$
I_{d+e}^{\mu, B M}=\bar{u}\left(p^{\prime}\right) \tilde{F}(q) \int \frac{\mathrm{d}^{4} k}{(2 \pi)^{4}} \frac{\tilde{F}(k)^{2}}{D_{M}(k)}\left\{\not k \gamma_{5} \frac{1}{\not p^{\prime}-\not k-m_{B}} \gamma^{\mu} \gamma_{5}+\gamma^{\mu} \gamma_{5} \frac{1}{\not p-\not k-m_{B}} \not k \gamma_{5}\right\} u(p) .
$$

Figures 1(f) and $1(\mathrm{~g})$ are the additional diagrams that are generated from the expansion of the gauge link. The contributions of these two diagrams are expressed as

$$
\begin{aligned}
& \Gamma_{f+g, u}^{\mu}\left(\Sigma^{+}\right)=\frac{D^{2}+3 F^{2}}{6 f^{2}} I_{f+g}^{\mu, \Sigma \pi}, \\
& \Gamma_{f+g, d}^{\mu}\left(\Sigma^{+}\right)=\frac{D^{2}}{3 f^{2}} I_{f+g}^{\mu, \Lambda \pi}+\frac{F^{2}}{f^{2}} I_{f+g}^{\mu, \Sigma \pi}+\frac{(D-F)^{2}}{2 f^{2}} I_{f+g}^{\mu, N K}, \\
& \Gamma_{f+g, s}^{\mu}\left(\Sigma^{+}\right)=\frac{D^{2}+3 F^{2}}{3 f^{2}} I_{f+g}^{\mu, \Xi K},
\end{aligned}
$$

where

$$
\begin{aligned}
I_{f+g}^{\mu, B M}= & \bar{u}\left(p^{\prime}\right) \tilde{F}(q) \int \frac{\mathrm{d}^{4} k}{(2 \pi)^{4}} \frac{\tilde{F}(k)}{D_{M}(k)}\left\{\frac{(2 k-q)^{\mu}}{2 k q-q^{2}}[\tilde{F}(k-q)-\tilde{F}(k)] \not \gamma_{5} \frac{1}{\not p^{\prime}-\not k-m_{B}}(-\not k+q) \gamma_{5}\right. \\
& \left.+\frac{(2 k+q)^{\mu}}{2 k q+q^{2}}[\tilde{F}(k+q)-\tilde{F}(k)](\not k+q) \gamma_{5} \frac{1}{\not p-\not k-m_{B}} \not k \gamma_{5}\right\} u(p) .
\end{aligned}
$$

Now we show the expressions of one loop integrals for decuplet intermediate states. The contributions for Fig. 1(h) can be written as 


$$
\begin{aligned}
& \Gamma_{h, u}^{\mu}\left(\Sigma^{+}\right)=\frac{\mathcal{C}^{2}}{24 f^{2}} I_{h}^{\mu, \Sigma^{*} \pi}+\frac{\mathcal{C}^{2}}{6 f^{2}} I_{h}^{\mu, \Delta K}, \\
& \Gamma_{h, d}^{\mu}\left(\Sigma^{+}\right)=\frac{\mathcal{C}^{2}}{12 f^{2}} I_{h}^{\mu, \Sigma^{*} \pi}+\frac{\mathcal{C}^{2}}{6 f^{2}} I_{h}^{\mu, \Delta K}, \\
& \Gamma_{h, s}^{\mu}\left(\Sigma^{+}\right)=\frac{\mathcal{C}^{2}}{12 f^{2}} I_{h}^{\mu, \Xi^{*} K},
\end{aligned}
$$

where the integral $I_{h}^{\mu, T M}$ is expressed as

$$
\begin{aligned}
I_{h}^{\mu, T M}= & \bar{u}\left(p^{\prime}\right) \tilde{F}(q) \int \frac{\mathrm{d}^{4} k}{(2 \pi)^{4}} \frac{\tilde{F}(q+k) \tilde{F}(k)}{D_{M}(k)} \frac{2(k+q)^{\mu}}{D_{M}(q+k)} \\
& \times\left((k+q)^{\sigma}+z(\not k+q) \gamma^{\sigma}\right) \frac{1}{\not p-\not k-m_{T}} S_{\sigma \rho}(p-k)\left(-k^{\rho}-z \gamma^{\rho} \not k\right) u(p) .
\end{aligned}
$$

$m_{T}$ is the mass of the decuplet intermediate state and $S_{\sigma \rho}(p)$ is expressed as

$$
S_{\sigma \rho}(p)=-g_{\sigma \rho}+\frac{\gamma_{\sigma} \gamma_{\rho}}{3}+\frac{p_{\sigma} p_{\rho}}{3 m_{T}^{2}}+\frac{\gamma_{\sigma} p_{\rho}-\gamma_{\rho} p_{\sigma}}{3 m_{T}} .
$$

The contributions for Fig. 1(i) are written as

$$
\begin{aligned}
& \Gamma_{i, u}^{\mu}\left(\Sigma^{+}\right)=\frac{\mathcal{C}^{2}\left(\left(c_{1}+3 c_{2}+3\right) Q^{2}+12 m_{\Sigma^{*}}^{2}\right)}{72 f^{2}\left(4 m_{\Sigma^{*}}^{2}+Q^{2}\right)} I_{i}^{\mu, \Sigma^{*} \pi}+\frac{\mathcal{C}^{2}\left(\left(c_{1}+3 c_{2}+3\right) Q^{2}+12 m_{\Delta}^{2}\right)}{18 f^{2}\left(4 m_{\Delta}^{2}+Q^{2}\right)} I_{i}^{\mu, \Delta K}, \\
& \Gamma_{i, d}^{\mu}\left(\Sigma^{+}\right)=\frac{\mathcal{C}^{2}\left(\left(c_{1}+3 c_{2}+3\right) Q^{2}+12 m_{\Sigma^{*}}^{2}\right)}{36 f^{2}\left(4 m_{\Sigma^{*}}^{2}+Q^{2}\right)} I_{i}^{\mu, \Sigma^{*} \pi}+\frac{\mathcal{C}^{2}\left(\left(c_{1}+3 c_{2}+3\right) Q^{2}+12 m_{\Delta}^{2}\right)}{18 f^{2}\left(4 m_{\Delta}^{2}+Q^{2}\right)} I_{i}^{\mu, \Delta K}, \\
& \Gamma_{i, s}^{\mu}\left(\Sigma^{+}\right)=\frac{\mathcal{C}^{2}\left(\left(c_{1}+3 c_{2}+3\right) Q^{2}+12 m_{\Xi^{*}}^{2}\right)}{36 f^{2}\left(4 m_{\Xi^{*}}^{2}+Q^{2}\right)} I_{i}^{\mu, \Xi^{*} K},
\end{aligned}
$$

where the integral $I_{i}^{\mu, T M}$ is written as

$$
\begin{aligned}
I_{i}^{\mu, T M}= & \bar{u}\left(p^{\prime}\right) \tilde{F}(q) \int \frac{\mathrm{d}^{4} k}{(2 \pi)^{4}} \frac{\tilde{F}(k)^{2}}{D_{M}(k)}\left(k^{\sigma}+z \not k \gamma^{\sigma}\right) \\
& \times \frac{1}{\not p^{\prime}-\not k-m_{T}} S_{\sigma \alpha}\left(p^{\prime}-k\right) \gamma^{\alpha \beta \mu} \frac{1}{\not p-\not k-m_{T}} S_{\beta \rho}(p-k)\left(k^{\rho}+z \gamma^{\rho} \not k\right) u(p) .
\end{aligned}
$$

The contributions for Fig. 1(j) are written as

$$
\begin{aligned}
& \Gamma_{j, u}^{\mu}\left(\Sigma^{+}\right)=-\frac{i\left(c_{1}+3 c_{2}\right) \mathcal{C}^{2} m_{\Sigma^{*}}}{36 f^{2}\left(4 m_{\Sigma^{*}}^{2}+Q^{2}\right)} I_{j}^{\mu, \Sigma^{*} \pi}-\frac{i\left(c_{1}+3 c_{2}\right) \mathcal{C}^{2} m_{\Delta}}{9 f^{2}\left(4 m_{\Delta}^{2}+Q^{2}\right)} I_{j}^{\mu, \Delta K}, \\
& \Gamma_{j, d}^{\mu}\left(\Sigma^{+}\right)=-\frac{i\left(c_{1}+3 c_{2}\right) \mathcal{C}^{2} m_{\Sigma^{*}}}{18 f^{2}\left(4 m_{\Sigma^{*}}^{2}+Q^{2}\right)} I_{j}^{\mu, \Sigma^{*} \pi}-\frac{i\left(c_{1}+3 c_{2}\right) \mathcal{C}^{2} m_{\Delta}}{9 f^{2}\left(4 m_{\Delta}^{2}+Q^{2}\right)} I_{j}^{\mu, \Delta K}, \\
& \Gamma_{j, s}^{\mu}\left(\Sigma^{+}\right)=-\frac{i\left(c_{1}+3 c_{2}\right) \mathcal{C}^{2} m_{\Xi^{*}}}{18 f^{2}\left(4 m_{\Xi^{*}}^{2}+Q^{2}\right)} I_{j}^{\mu, \Xi^{*} K}
\end{aligned}
$$

where the integral $I_{j}^{\mu, T M}$ is expressed as 


$$
\begin{aligned}
I_{j}^{\mu, T M}= & \bar{u}\left(p^{\prime}\right) \tilde{F}(q) \int \frac{\mathrm{d}^{4} k}{(2 \pi)^{4}} \frac{\tilde{F}(k)^{2}}{D_{M}(k)}\left(k^{\sigma}+z \not k \gamma^{\sigma}\right) \\
& \times \frac{1}{\not p^{\prime}-\not k-m_{T}} S_{\sigma \nu}\left(p^{\prime}-k\right) i \sigma^{\mu \lambda} q_{\lambda} \frac{1}{\not p-\not k-m_{T}} S^{\nu \rho}(p-k)\left(k_{\rho}+z \gamma_{\rho} \not k\right) u(p) .
\end{aligned}
$$

The contributions for the intermediate octet-decuplet transition diagrams Figs. 1(k) and 1(l) are expressed as

$$
\begin{aligned}
\Gamma_{k+l, u}^{\mu}\left(\Sigma^{+}\right) & =\frac{c_{1} \mathcal{C}(D-F)}{24 f^{2} m_{\Sigma}} I_{k+l}^{\mu, \Sigma^{*} \Sigma \pi}, \\
\Gamma_{k+l, d}^{\mu}\left(\Sigma^{+}\right) & =\frac{c_{1} \mathcal{C} D}{12 f^{2} m_{\Lambda}} I_{k+l}^{\mu, \Sigma^{*} \Lambda \pi}-\frac{c_{1} \mathcal{C} F}{12 f^{2} m_{\Sigma}} I_{k+l}^{\mu, \Sigma^{*} \Sigma \pi}+\frac{c_{1} \mathcal{C}(D-F)}{6 f^{2} m_{N}} I_{k+l}^{\mu, \Delta N \pi}, \\
\Gamma_{k+l, s}^{\mu}\left(\Sigma^{+}\right) & =\frac{c_{1} \mathcal{C}(D-F)}{12 f^{2} m_{\Xi}} I_{k+l}^{\mu, \Xi^{*} \Xi K},
\end{aligned}
$$

where the integral $I_{k+l}^{\mu, T B M}$ is written as

$$
\begin{aligned}
I_{k+l}^{\mu, T B M}= & \bar{u}\left(p^{\prime}\right) \tilde{F}(q) \int \frac{\mathrm{d}^{4} k}{(2 \pi)^{4}} \frac{\tilde{F}(k)^{2}}{D_{M}(k)}\left\{\not k \gamma_{5} \frac{1}{\not p^{\prime}-\not k-m_{B}}\left(-\not \gamma_{5}\right) \frac{1}{\not p-\not k-m_{T}} S^{\mu \rho}(p-k)\left(k_{\rho}+z \gamma_{\rho} \not k\right)\right. \\
& +\not k \gamma_{5} \frac{1}{\not p^{\prime}-\not k-m_{B}} \gamma^{\mu} \gamma_{5} q_{\nu} \frac{1}{\not p-\not k-m_{T}} S^{\nu \rho}(p-k)\left(k_{\rho}+z \gamma_{\rho} \not k\right) \\
& +\left(k_{\nu}+z \not k \gamma_{\nu}\right) \frac{1}{\not p^{\prime}-\not k-m_{T}} S^{\nu \rho}\left(p^{\prime}-k\right)\left(-q_{\rho} \gamma^{\mu} \gamma_{5}\right) \frac{1}{\not p-\not k-m_{B}} \not \gamma_{5} \\
& \left.+\left(k_{\nu}+z \not k \gamma_{\nu}\right) \frac{1}{\not p^{\prime}-\not k-m_{T}} S^{\nu \mu}\left(p^{\prime}-k\right) \not \gamma_{5} \frac{1}{\not p-\not k-m_{B}} \not k \gamma_{5}\right\} u(p) .
\end{aligned}
$$

The contributions for the Kroll-Ruderman diagrams Figs. 1(m) and 1(n) are written as

$$
\begin{aligned}
& \Gamma_{m+n, u}^{\mu}\left(\Sigma^{+}\right)=\frac{\mathcal{C}^{2}}{24 f^{2}} I_{m+n}^{\mu, \Sigma^{*} \pi}+\frac{\mathcal{C}^{2}}{6 f^{2}} I_{m+n}^{\mu, \Delta K}, \\
& \Gamma_{m+n, d}^{\mu}\left(\Sigma^{+}\right)=\frac{\mathcal{C}^{2}}{12 f^{2}} I_{m+n}^{\mu, \Sigma^{*} \pi}+\frac{\mathcal{C}^{2}}{6 f^{2}} I_{m+n}^{\mu, \Delta K}, \\
& \Gamma_{m+n, s}^{\mu}\left(\Sigma^{+}\right)=\frac{\mathcal{C}^{2}}{12 f^{2}} I_{m+n}^{\mu, \Xi^{*} K},
\end{aligned}
$$

where the integral $I_{m+n}^{\mu, T M}$ is written as

$$
\begin{aligned}
I_{m+n}^{\mu, T M}= & \bar{u}\left(p^{\prime}\right) \tilde{F}(q) \int \frac{\mathrm{d}^{4} k}{(2 \pi)^{4}} \frac{\tilde{F}(k)^{2}}{D_{M}(k)}\left\{\left(k_{\sigma}+z \not k \gamma_{\sigma}\right) \frac{1}{\not p^{\prime}-\not k-m_{T}} S^{\sigma \rho}\left(p^{\prime}-k\right)\left(g_{\rho}^{\mu}+z \gamma_{\rho} \gamma^{\mu}\right)\right. \\
& \left.+\left(g_{\sigma}^{\mu}+z \gamma^{\mu} \gamma_{\sigma}\right) \frac{1}{\not p-\not k-m_{T}} S^{\sigma \rho}(p-k)\left(k_{\rho}+z \gamma_{\rho} \not k\right)\right\} u(p) .
\end{aligned}
$$

Finally, the contributions for the additional diagrams with intermediate decuplet states Figs. 1(o) and 1(p) are expressed as

$$
\Gamma_{o+p, u}^{\mu}\left(\Sigma^{+}\right)=\frac{\mathcal{C}^{2}}{24 f^{2}} I_{o+p}^{\mu, \Sigma^{*} \pi}+\frac{\mathcal{C}^{2}}{6 f^{2}} I_{o+p}^{\mu, \Delta K},
$$




$$
\begin{aligned}
& \Gamma_{o+p, d}^{\mu}\left(\Sigma^{+}\right)=\frac{\mathcal{C}^{2}}{12 f^{2}} I_{o+p}^{\mu, \Sigma^{*} \pi}+\frac{\mathcal{C}^{2}}{6 f^{2}} I_{o+p}^{\mu, \Delta K}, \\
& \Gamma_{o+p, s}^{\mu}\left(\Sigma^{+}\right)=\frac{\mathcal{C}^{2}}{12 f^{2}} I_{o+p}^{\mu, \Xi^{*} K},
\end{aligned}
$$

where the integral $I_{o+p}^{\mu, T M}$ is written as

$$
\begin{aligned}
I_{o+p}^{\mu, T M}= & \bar{u}\left(p^{\prime}\right) \tilde{F}(q) \int \frac{\mathrm{d}^{4} k}{(2 \pi)^{4}} \frac{\tilde{F}(k)}{D_{M}(k)} \\
& \times\left\{\frac{(-2 k+q)^{\mu}}{-2 k q+q^{2}}(\tilde{F}(k-q)-\tilde{F}(k))\left(k_{\sigma}+z \not k \gamma_{\sigma}\right) \frac{1}{\not p^{\prime}-\not k-m_{T}} S^{\sigma \rho}\left(p^{\prime}-k\right)\left((k-q)_{\rho}+z \gamma_{\rho}(\not k-q)\right)\right. \\
& \left.+\frac{(2 k+q)^{\mu}}{2 k q+q^{2}}(\tilde{F}(k+q)-\tilde{F}(k))\left((k+q)_{\sigma}+z(\not k+q) \gamma_{\sigma}\right) \frac{1}{\not p-\not k-m_{T}} S^{\sigma \rho}(p-k)\left(k_{\rho}+z \gamma_{\rho} \not k\right)\right\} u(p) .
\end{aligned}
$$

Using Package-X [49] to simplify the loop integral, we have gathered the results for the electromagnetic form factors.

[1] D. T. Spayde et al. (SAMPLE Collaboration), The strange quark contribution to the proton's magnetic moment, Phys. Lett. B 583, 79 (2004).

[2] F. E. Maas et al. (A4 Collaboration), Measurement of Strange Quark Contributions to the Nucleon's Form-Factors at $Q^{2}=0.230(\mathrm{GeV} / \mathrm{c})^{2}$, Phys. Rev. Lett. 93, 022002 (2004).

[3] F. E. Maas et al., Evidence for Strange Quark Contributions to the Nucleon's Form-Factors at $q^{2}=0.108(\mathrm{GeV} / \mathrm{c})^{2}$, Phys. Rev. Lett. 94, 152001 (2005).

[4] D. S. Armstrong et al. (G0 Collaboration), Strange Quark Contributions to Parity-Violating Asymmetries in the Forward G0 Electron-Proton Scattering Experiment, Phys. Rev. Lett. 95, 092001 (2005).

[5] D. Androic et al. (G0 Collaboration), Strange Quark Contributions to Parity-Violating Asymmetries in the Backward Angle G0 Electron Scattering Experiment, Phys. Rev. Lett. 104, 012001 (2010).

[6] K. A. Aniol et al. (HAPPEX Collaboration), Parity violating electroweak asymmetry in polarized $-\vec{e} p$ scattering, Phys. Rev. C 69, 065501 (2004).

[7] K. A. Aniol et al. (HAPPEX Collaboration), Constraints on the nucleon strange form-factors at $Q^{2} \sim 0.1-\mathrm{GeV}^{2}$, Phys. Lett. B 635, 275 (2006).

[8] A. Acha et al. (HAPPEX Collaboration), Precision Measurements of the Nucleon Strange Form Factors at $Q^{2} \sim 0.1-\mathrm{GeV}^{2}$, Phys. Rev. Lett. 98, 032301 (2007).

[9] Z. Ahmed et al. (HAPPEX Collaboration), New Precision Limit on the Strange Vector Form Factors of the Proton, Phys. Rev. Lett. 108, 102001 (2012).

[10] R. D. Young, J. Roche, R. D. Carlini, and A. W. Thomas, Extracting Nucleon Strange and Anapole form Factors from World Data, Phys. Rev. Lett. 97, 102002 (2006).
[11] R. González-Jiménez, J. A. Caballero, and T. W. Donnelly, Global analysis of parity-violating asymmetry data for elastic electron scattering, Phys. Rev. D 90, 033002 (2014).

[12] R. L. Jaffe, Stranger than fiction: The strangeness radius and magnetic moment of the nucleon, Phys. Lett. B 229, 275 (1989).

[13] N. W. Park, J. Schechter, and H. Weigel, Electromagnetic, axial and strange currents in the Skyrme model: Effects of symmetry breaking, Phys. Rev. D 43, 869 (1991).

[14] T. D. Cohen, H. Forkel, and M. Nielsen, Just how strange? Loops, poles and the strangeness radius of the nucleon, Phys. Lett. B 316, 1 (1993).

[15] M. J. Musolf and M. Burkardt, Stranger still: Kaon loops and strange quark matrix elements of the nucleon, Z. Phys. C 61, 433 (1994).

[16] H. Weigel, A. Abada, R. Alkofer, and H. Reinhardt, On the strange vector form-factors of the nucleon in the NJL soliton model, Phys. Lett. B 353, 20 (1995).

[17] H. W. Hammer, U.-G. Meissner, and D. Drechsel, The strangeness radius and magnetic moment of the nucleon revisited, Phys. Lett. B 367, 323 (1996).

[18] S.-T. Hong, B.-Y. Park, and D.-P. Min, Strange form-factors of baryons, Phys. Lett. B 414, 229 (1997).

[19] L. Hannelius, D. O. Riska, and L. Y. Glozman, The strangeness magnetic moment of the proton in the chiral quark model, Nucl. Phys. A665, 353 (2000).

[20] A. Silva, H.-C. Kim, and K. Goeke, Strange form-factors in the context of SAMPLE, HAPPEX, and A4 experiments, Phys. Rev. D 65, 014016 (2001); 66, 039902(E) (2002).

[21] V.E. Lyubovitskij, P. Wang, T. Gutsche, and A. Faessler, Strange nucleon form-factors in the perturbative chiral quark model, Phys. Rev. C 66, 055204 (2002). 
[22] B. S. Zou and D. O. Riska, $s \bar{s}$ Component of the Proton and the Strangeness Magnetic Moment, Phys. Rev. Lett. 95, 072001 (2005).

[23] M. J. Musolf and H. Ito, Chiral symmetry and the nucleon's vector strangeness form-factors, Phys. Rev. C 55, 3066 (1997).

[24] T. R. Hemmert, B. Kubis, and U.-G. Meissner, Strange chiral nucleon form-factors, Phys. Rev. C 60, 045501 (1999).

[25] B. Kubis, Strange form-factors and chiral perturbation theory, Eur. Phys. J. A 24, 97 (2005).

[26] T. Fuchs, J. Gegelia, and S. Scherer, Electromagnetic form factors of the nucleon in chiral perturbation theory, J. Phys. G 30, 1407 (2004).

[27] B. Kubis and U.-G. Meissner, Low-energy analysis of the nucleon electromagnetic form factors, Nucl. Phys. A679, 698 (2001).

[28] C. R. Allton, W. Armour, D. B. Leinweber, A. W. Thomas, and R.D. Young, Chiral and continuum extrapolation of partially-quenched lattice results, Phys. Lett. B 628, 125 (2005).

[29] P. Wang, D. B. Leinweber, A. W. Thomas, and R. D. Young, Chiral extrapolation of nucleon magnetic form factors, Phys. Rev. D 75, 073012 (2007).

[30] H. Li, P. Wang, D. B. Leinweber, and A. W. Thomas, Spin of the proton in chiral effective field theory, Phys. Rev. C 93, 045203 (2016).

[31] P. Wang, D. B. Leinweber, and A. W. Thomas, Strange magnetic form factor of the nucleon in a chiral effective model at next to leading order, Phys. Rev. D 89, 033008 (2014).

[32] P. Wang, D. B. Leinweber, A. W. Thomas, and R. D. Young, Strange magnetic form factor of the proton at $Q^{2}=$ $0.23 \mathrm{GeV}^{2}$, Phys. Rev. C 79, 065202 (2009).

[33] F. He and P. Wang, Nucleon electromagnetic form factors with a nonlocal chiral effective lagrangian, Phys. Rev. D 97, 036007 (2018).

[34] F. He and P. Wang, Strange form factors of the nucleon with a nonlocal chiral effective lagrangian, Phys. Rev. D 98, 036007 (2018).

[35] M. Yang and P. Wang, Electromagnetic form factors of octet baryons with the nonlocal chiral effective theory, preceding paper, Phys. Rev. D 102, 056024 (2020).
[36] Y. Salamu, C.-R. Ji, W. Melnitchouk, A. W. Thomas, and P. Wang, Parton distributions from nonlocal chiral SU(3) effective theory: Splitting functions, Phys. Rev. D 99, 014041 (2019).

[37] Y. Salamu, C.-R. Ji, W. Melnitchouk, A. W. Thomas, P. Wang, and X. G. Wang, Parton distributions from nonlocal chiral SU(3) effective theory: Flavor asymmetries, Phys. Rev. D 100, 094026 (2019).

[38] M.-Y. Yang and P. Wang, Sea quark contributions to nucleon electromagnetic form factors with the nonlocal chiral effective Lagrangian, Chin. Phys. C 44, 053101 (2020).

[39] R. S. Sufian, Y.-B. Yang, J. Liang, T. Draper, and K. -F. Liu, Sea quarks contribution to the nucleon magnetic moment and charge radius at the physical point, Phys. Rev. D 96, 114504 (2017).

[40] C. Alexandrou, M. Constantinou, K. Hadjiyiannakou, K. Jansen, C. Kallidonis, G. Koutsou, and A. Vaquero AvilésCasco, Strange nucleon electromagnetic form factors from lattice QCD, Phys. Rev. D 97, 094504 (2018).

[41] E. E. Jenkins, Baryon masses in chiral perturbation theory, Nucl. Phys. B368, 190 (1992).

[42] E. E. Jenkins, M. E. Luke, A. V. Manohar, and M. J. Savage, Chiral perturbation theory analysis of the baryon magnetic moments, Phys. Lett. B 302, 482 (1993).

[43] P. Wang, Solid quantization for nonpoint particles, Can. J. Phys. 92, 25 (2014).

[44] D. B. Leinweber, Quark contributions to baryon magnetic moments in full, quenched, and partially quenched QCD, Phys. Rev. D 69, 014005 (2004).

[45] C. W. Bernard and M. F. L. Golterman, Chiral perturbation theory for the quenched approximation of QCD, Phys. Rev. D 46, 853 (1992).

[46] B. Borasoy and U.-G. Meissner, Chiral expansion of baryon masses and $\sigma$-terms, Ann. Phys. (N.Y.) 254, 192 (1997).

[47] L. M. Nath, B. Etemadi, and J. D. Kimel, Uniqueness of the interaction involving spin- $\frac{3}{2}$ particles, Phys. Rev. D 3, 2153 (1971).

[48] P. Zyla et al. (Particle Data Group), Review of particle physics, Prog. Theor. Exp. Phys. 2020, 083 C01 (2020).

[49] H. H. Patel, Package-X: A Mathematica package for the analytic calculation of one-loop integrals, Comput. Phys. Commun. 197, 276 (2015). 